\title{
Joint Polar Satellite System (JPSS) Instrument Transition to NASA and Development Status
}

\author{
Christopher W. Brann \\ Integrity Applications Inc., USA
}

\begin{abstract}
The Joint Polar Satellite System (JPSS) Program is a cooperative program between the National Aeronautics and Space Agency (NASA) and the National Oceanic and Atmospheric Administration (NOAA) to design, develop, and fly the next suite of US civilian polar orbiting environmental sensing instruments. The JPSS Program is a product of the restructuring of the National Polar-orbiting Operational Environmental Satellite System (NPOESS) Program, which occurred in 2010. With the transition to NASA, the JPSS instruments have undergone significant review with numerous updates to the designs as well as made significant progress toward delivering a superior capability to the Nation. This paper will discuss the program transition as it relates to the instruments and the associated transition review efforts, key findings, important changes to the instruments for JPSS and their current development status. The VIIRS instrument will be presented separately.
\end{abstract}

Keywords: JPSS, Climate, VIIRS, CrIS, OMPS, ATMS, CERES, Weather

\section{INTRODUCTION}

The newly created JPSS Program will satisfy the NOAA requirements for collection of global multi-spectral radiometry and other specialized meteorological, oceanographic, and solar-geophysical data via remote sensing of land, sea, and atmospheric data. This data is necessary for meteorological and global climate observation and tracking. The first JPSS satellite is scheduled for launch in 2016. Each JPSS Flight Satellite is comprised of five instruments, Advanced Technology Microwave Sounder (ATMS), Cloud and Earth's Radiant Energy System (CERES), Cross-track Infrared Sounder (CrIS), Ozone Mapping and Profiler Suite (OMPS), and Visible/Infrared Imager Radiometer Suite (VIIRS).

The Goddard Space Flight Center (GSFC) is responsible for overall implementation of the JPSS Flight Mission. GSFC has issued contracts for the spacecraft development with Ball Aerospace Technology Corporation (BATC); to Raytheon Space and Airborne Systems (RSAS) for VIIRS; to BATC for OMPS; to Exelis for CrIS; and to Northrop Grumman Electronic Systems (NGES) for ATMS. The NASA Langley Research Center (LaRC) is responsible for CERES and has issued a contract to Northrop Grumman Aerospace Systems (NGAS) for CERES. The Kennedy Space Center is responsible for launch services.

The ATMS instrument is a total power cross-track scanning radiometer with 22 channels, providing sounding observations needed to retrieve profiles of atmospheric temperature and moisture for civilian operational weather forecasting as well as continuity of these measurements for climate monitoring purposes. The CrIS instrument consists of a Fourier transform spectrometer with 1305 spectral channels that produces high-resolution, three-dimensional temperature, pressure, and moisture profiles. The VIIRS instrument is a scanning radiometer which collects visible and infrared imagery and radiometric measurements of the land, atmosphere, cryosphere, and oceans. Additionally, VIIRS provides data regarding cloud properties which include the amount, height, thickness, particle size, and phase of clouds using simultaneous measurements by other instruments. With respect to climate, OMPS is an advanced suite of two hyper-spectral instruments that extends the 25-plus year total-ozone and ozone-profile records. CERES is a threechannel radiometer that measures both solar-reflected and Earth-emitted radiation from the top of the atmosphere to the surface.

The 2010 launch of the Suomi National Polar-orbiting Partnership (NPP) satellite consists of the same five instruments planned for operations with the JPSS program. NPP was initially intended to not only provide valuable science, but also 
provide critical lessons learned and risk reduction for the follow on instruments. In order to effectively transition JPSS and its instrument acquisition responsibilities to NASA, the JPSS instrument designs, requirements and development processes were thoroughly reviewed. Several changes were made to both improve the performance of the instruments as well as their reliability and manufacturability.

\section{INSTRUMENT TRANSITION TO NASA}

During the summer of 2010, a massive cooperative effort between the US Air Force, NOAA and NASA was undertaken to efficiently transition the Flight and Ground elements of the NPOESS program to NASA. NPOESS was administered via a US Air Force contract to the Prime Contractor Northrop Grumman Aerospace Systems (NGAS). Although the US Government began the initial instrument procurements on unique contracts, by 2010 each of the instrument contracts were a subcontract to NGAS and being developed to interface to the Northrop Grumman NPOESS spacecraft. Thus, each contract had requirements which were not consistent with the JPSS spacecraft or with many Goddard Space Flight Center (GSFC) standards.

Reviews conducted by subject matter experts included a comprehensive assessment of the instrument against the GSFC Environmental Verification Standard (GEVS); GSFC GOLD Rules (Rules for the Design, Development, Verification, and Operation of Flight Systems); GPR 7120.7, Schedule Margins and Budget Reserves; 7120.4 NASA Engineering and Management Policy; Mission Assurance Requirements; all documented risks; NPP waivers applicable to JPSS; and any potential upgrades and improvements.

\subsection{Residual Risks}

The JPSS Instrument Management team reviewed the residual risks associated with each NPP instrument, determined whether those risks were applicable to JPSS and if so how to mitigate those risks for the new program. The team reviewed all NPP/NPOESS instrument related Government independent review assessments, risk databases and pre-ship review packages. 103 residual risks were identified which were applicable to JPSS. Of those risks $67 \%$ of the NPP Residual Risks are now retired or closed for JPSS as a result of mitigating design changes, specification updates or agreed to dispositions. The remaining risks are actively tracked for JPSS Instruments. Two realized risks were related to the OMPS Single Board Computer (SBC) and one for VIIRS related to contamination due to paint flaking on the cryocooler which were realized. Both of those issues are now resolved. The top risk areas identified from this review are shown below in Figure 1. The arrows reflect those NPP-era risks assessed based on their planned mitigations for JPSS.

As shown five of the risks have been projected as retired and the five remaining risks are being addressed. The team has reviewed the various life testing and determined the risk to be acceptable once additional inspections are complete this summer on OMPS. JPSS was concerned that the CrIS Focal Plane Arrays (FPA) may have some temperature dependencies based on experience from the NPP build. These FPAs were successfully retested over temperature retiring this risk. White wires are typically circuit card fixes after the card is built and represent a potential reliability concern. All circuit cards were inspected by NASA mission assurance experts from which decisions were made on whether to redesign the circuit cards to eliminate the white wires or use as is. For the VIIRS instrument, all circuit cards were redesigned to remove the need for such wires; for CrIS and OMPS, those circuit cards with the most modifications are being redesigned to remove the white wires and replace $\mathrm{cPCI}$ connectors with higher reliability connectors. A lack of spare hardware is a concern across all instruments; therefore, JPSS has applied funding to procure a prioritized set of critical spares significantly reducing this risk. The remaining risks are being addressed through parts screening and improvements to the test program. 


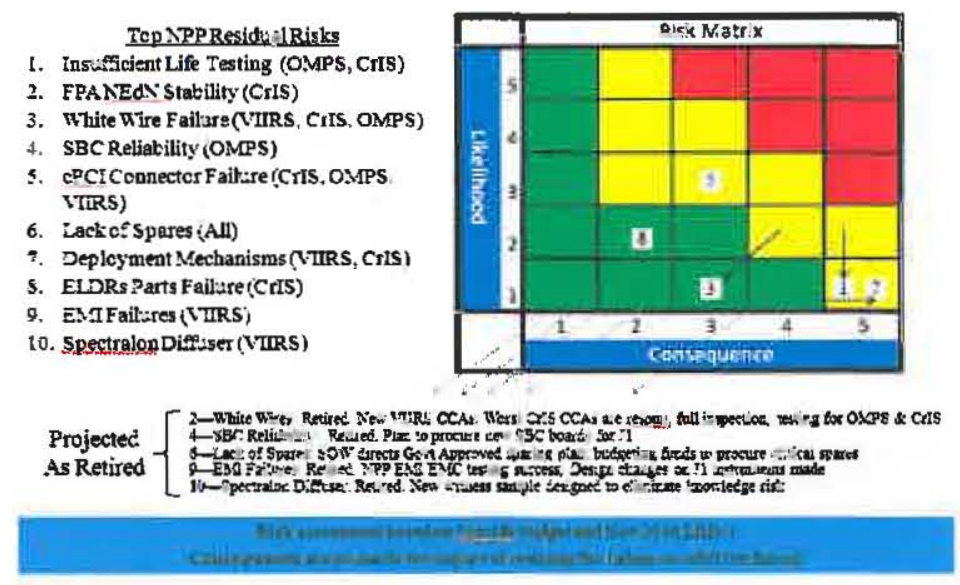

Figure 1. Risk Assessment

\subsection{Mission Assurance}

The Mission Assurance Requirements reviews resulted in the development of the Instrument Mission Assurance Requirements (IMAR) document and an associated compliance plan for each instrument on JPSS. This new requirement ensures that the instrument is being built to expected NASA mission assurance practices, that any deviations are well understood, documented and approved by mission assurance experts improving the reliability of the mission as a whole.

The major finding from this effort was related to the use of cPCI connectors and white wires; both of which have been completely analyzed and assessed by subject matter experts and the dispositions for JPSS agreed to. cPCI connectors are a type of commercial tuning fork connectors commonly used in aerospace settings; however, in the past the connections have failed due to erosion of the normally thin gold plating and the subsequent build up of oxidation. The plating can be worn away due to mate/demate cycles or operational vibrations. The OMPS instrument has redesigned its backplane, single board computer circuit cards and Processor Interface circuit cards to address identified parts concerns and to replace the lower reliability commercial connectors with NASA approved Hypertronix connectors. Both VIIRS and CrIS instruments were analyzed for dynamics at the connectors and for connector metallurgy. Both were determined acceptable for JPSS based on their use of appropriately modified $\mathrm{cPCI}$ connectors which have greater gold plating thickness and hardness.

The CrIS instrument will have redesigned circuit cards to remove the need for white wires. Several mandatory inspection points were added to each of the instrument contracts enabling NASA mission assurance experts to inspect and approve the hardware as it proceeds through its build and test. In addition to the hardware, all field programmable gate array (FPGA) designs were reviewed by NASA subject matter experts and approved for use. Based on lessons learned from NPP and the lack of long term supportability for MIL-STD-1394 "Firewire" technology, JPSS is replacing the 1394 data bus with the SpaceWire architecture. This will improve the reliability of the system and simplify the designs. This change affects CrIS, VIIRS and the Spacecraft only. The other instruments use a MIL-STD-1553 communication architecture.

The Flight Software is being reviewed by NASA Software Mission Assurance experts for potential risks. It is expected that some modifications will be required as a result of the software reviews and the Spacewire change. VIIRS is modifying its flight software to improve reliability based on lessons learned from NPP operations. One example is the addition of a software monitoring routine which enables a soft reset were the VIIRS single board computer to lock up. Other modifications include improved error detection and correction algorithms to improve operational reliability.

\subsection{Waivers}

The JPSS team reviewed all waivers from NPP. 89 NPP waivers have been addressed for JPSS through design changes or updates to the requirements based on lessons learned from NPP and are no longer applicable to JPSS. The remaining 
waivers represent low risk on JPSS with respect to performance degradations from that witnessed on NPP and are awaiting test results. Currently there are no expected waivers that degrade performance from that of NPP; nearly all waivers expect to reflect an improvement over NPP performance due to improved processes and lessons learned from NPP.

The ATMS instrument has one potential waiver for not meeting the specified 95\% Antenna Beam Efficiencies at 183.3 $\mathrm{GHz}$ and $190.3 \mathrm{GHz}$. Extensive improvements to the test program were made to better characterize the beam efficiency and polarization effects from that done on NPP. The engineering test data showed that the $95 \%$ beam efficiency was met; however, those tests were not conducted as verification tests. As a result a waiver is still required. The improved knowledge will likely improve performance over NPP in this area. In addition to the beam efficiency test improvements, beam pointing accuracy and polarization testing have been significantly changed to improve our knowledge and further reduce uncertainty. The JPSS team is evaluating the test data of the antenna pointing knowledge and polarization tests. No waivers have been accepted by JPSS for the ATMS instrument. The conclusion is that the failed test data was due to test methodology and not antenna performance. Using an alternate test approach now shows that ATMS meets beam efficiency at all frequencies.

The CrIS instrument had 49 waivers approved on NPP. On JPSS, 27 have been resolved through simple changes to the interface specification; five have been resolved through performance requirements changes based on lessons learned from NPP; and 10 were resolved through design or test changes which includes some additional testing. There are seven waivers anticipated for JPSS-1 CrIS. We anticipate having similar NEdN performance as that seen on NPP. No waivers have been accepted by JPSS for the CrIS instrument.

Two waivers are anticipated regarding material out-gassing due to the use of small amounts of paints and adhesives. A waiver will be required for the cooler cover which has pin pullers that are not designed to be double shear and for the Isolation System Frangibolt fasteners which are not positive locking as required by MIL-A-83577B. A GOLD rules waiver will be needed to address our inability to "Test as You Fly" with respect to the Vibration Isolation System. It is not integrated with the instrument during thermal vacuum tests due to test chamber limitations. The CrIS circuit cards were built to MIL-STD-1547 which is inconsistent with GSFC's preferred standard EEE-INST-002. The circuit card inspections conducted by NASA experts ensures this risk is very low. Multiple semiconductors, when de-rated according to EEE-INST-002, are non-compliant; therefore a waiver will be required.

No waivers have been accepted by JPSS for the OMPS instrument. OMPS-NPP waivers were evaluated by the JPSS team to determine their applicability to JPSS. The majority of OMPS NPP waivers will be avoided as result of design and hardware fixes or requirements updates. Seven waivers are being submitted for OMPS to date. All are related to manufacturing process deviations and do not impact performance.

No waivers have been accepted by JPSS for the VIIRS instrument. 85 NPP waivers have been reviewed for applicability to the J1 instrument. 19 NPP waivers have been corrected. on JPSS via VIIRS design, test, or requirements changes. 39 NPP waivers are addressed on JPSS via specification and interface requirements changes. Of the 27 remaining concerns, all have been discussed extensively between Raytheon and JPSS to determine the desired path forward. In total there are 21 Performance requirements being addressed. None are expected to have an impact to the mission; however, further discussion is required to determine if they will be addressed with specification changes or require a JPSS waiver. There is an expected waiver related to the Absolute Reflectance Calibration Uncertainty due to out of band near field leakage for the three detectors on the Solar Diffuser Stability Monitor (SDSM). It is understood that periodic lunar calibrations will mitigate this shortcoming.

Specifically, there are nine requirements for various band-pairs and conditions related to band-to-band registration. The predicted J1 performance is superior to NPP due to design and alignment procedure improvements. There exist two waivers related to Spectral and Dynamic Range. The JPSS predicted performance is equivalent to NPP. The Short-Mid Wave Infrared (SMWIR), Long Wave Infrared (LWIR), and Day-Night Band (DNB) spectral characteristics are unchanged for JPSS. Regarding dynamic range, the I1 and M8 bands have limited margin with M1 having -1\%, again no change from NPP. 
JPSS performance is expected to be superior to NPP for radiometric characterization and calibration uncertainty due to various test improvements; however we do anticipate a need for lunar calibrations to overcome a recently discovered issue with the Solar Diffuser Stability Monitor. The SDSM performance on J1 will be the same as that on NPP which requires periodic lunar calibrations to reset our calibration knowledge and uncertainty. The other six waivers in this area are primarily an issue related to how the requirements are written. For example, the M11 Ltyp is required as equal to Lmin which is an unrealistic requirement. Relative radiometric response and characterization uncertainty are currently specified with respect to the measured noise, which penalizes VIIRS' very low noise floor. JPSS will address the requirements and specification clarity in future updates to the documents. There are two waivers related to stray light and one for reliability. The far-field stray light performance will be similar to that of NPP. The reliability waiver is also associated with mirror coatings. Due to limitations in industry-wide knowledge of degradation timelines for optical silver coatings, strict analytic verification is not possible. Raytheon has a well defined monitoring plan that will limit this risk; $\mathrm{J} 1$ performance will be similar to NPP.

A single waiver exists for data rates which will be addressed by the change to SpaceWire. No performance impacts are expected. Lastly there were four electromagnetic interference and/or compatibility (EMI/EMC) waivers on NPP. The JPSS team expects performance to be superior to NPP due to cable and connector design changes which should eliminate these issues.

The CERES instrument has completed its test program, but identified two performance issues that the team is working to resolve. These are related to the mirror attenuator mosaic's non-uniformity and the short wave calibration system's lamp and detector trending. There are currently six approved waivers for CERES and one in review. There is a waiver for the Flex Cable 90 degree solder joint that is not compliant with NASA 8739.1A (by design). A waiver exists documenting the acceptance of differences between the instrument contractor's standards and the NASA 8739 series Workmanship Standards. During build, there was polymeric rework performed on the Flex Cable Assemblies prior to government review and approval of rework processes which required a mission assurance waiver. There exist four small Cadmium Plated Hex Nuts in the CERES Encoders which require a waiver due to use of prohibited materials. A test related waiver was required due to improper post burn-in electrical testing of two logic gates. Lastly, a waiver exists for the LongWave (LW) Channel Filter. It does not fully meet spectral transmission requirements. The pending waiver is related to EMI. EMI radiated susceptibility exceeds limits in several narrow frequency bands over a bandwidth of $70 \mathrm{MHz}$; however, no degraded performance is expected.

\subsection{Upgrades and Improvements}

In addition to addressing residual risk, JPSS reviewed and prioritized a long list of proposed upgrades and improvements to the instruments. Most of the recommended design changes were associated with eliminating waivers and similar failures to those seen on NPP. Several changes were already in place or planned as corrective actions for NPP issues. Other changes proposed were based on reliability improvements, manufacturability improvements and minor performance enhancements.

\subsubsection{VIIRS}

For VIIRS numerous changes are being incorporated into the JPSS instrument. The three mirror anastigmat (TMA) in the rotating telescope assembly (RTA) will have new mirrors which change the coating to a Raytheon proprietary material which was used on some mirrors in the NPP instrument. Also a new coating process for the RTA will be used. These changes will significantly improve the optical performance of the instrument over temperature and reduce potential test delays during development. VIIRS J1 will have additional coating changes that will lower the reflectance with/between the solar diffuser. The RTA mirror mounting approach has been changed for the primary and secondary mirrors to improve robustness and add adjustability. These changes will lower the risk for J1 to meet existing optical performance requirements. The Visible Near-Infrared (VisNIR) Integrated Filter Assembly (IFA) has been redesigned to reduce optical crosstalk and out of band leakage within the IFA. A correction to the M2 bandwidth and center wavelength was made for the J1 IFA as well as requirements to address the NPP M2 saturation issues. A shimming of the four mirror assembly (FMA) Primary Mirror has been implemented to achieve overall system allocations. The need for Fold Mirror 2 (FM2) to have a small amount of power to compensate for focus as a function of temperature has been eliminated. The $\mathrm{J} 1$ mirror will be flat (unpowered) as originally designed. The Dichroic $1 \mathrm{NPP}$ required special 
mounting to orient the wedge; this is redesigned to address the mounting complexity. The Dichroic 2 on NPP has power due to coating stresses resulting in a different focus for SMWIR from other FPAs. The J1 Dichroic coating process has been redesigned allowing it to be flat (unpowered).

With respect to improvements in the electronics, several changes have been made. A different family of space flight FPGAs will be used that improve component reliability. As stated earlier, white wires have been designed out for JPSS resulting in fewer jumper wires on J1 improving reliability. The VisNIR Analog Signal Processor (ASP) FPGA circuit was changed to address dual gain switching noise. This resulted in reduced modification and jumper wires, some of which were added to NPP during troubleshooting. The Digital Backplane will have an increased trace length to account for a higher thermal load from the J1 Day-Night Band (DNB) heater. A Heater-Mechanism Assembly (HMA) inductor value was changed to support better thermal control of J1 DNB heater. Cable and connector modifications were made to address EMI outages and reduce outgassing contaminants. The Electronics Module (EM) housing surface treatment, three panels and four analog brackets were modified to eliminate any EMI paths to improve susceptibility performance. JPSS has modified the housing to prevent connector interference which was problematic on NPP. JPSS modified the Focal Plane Interface Electronics redundancy implementation to reduce electrostatic discharge (ESD) sensitivity improving reliability and reducing handling risks. A significant change to the JPSS interface is the replacement of the 1394 FireWire interface with the proven SpaceWire architecture for CrIS and VIIRS (other instruments use a 1553 bus interface). This change will improve reliability and simplify the overall observatory communication system design.

The JPSS VIIRS focal plane assemblies (FPA) will have some improvements over NPP as well. The VisNIR FPA has substrate and bonding improvements which are predicted to allow less dynamic crosstalk and provide better signal to noise ratio (SNR) performance. The Short-wave Medium-wave Infrared (SMWIR) FPA coating on the short wavelength detector changed to a more robust design to provide improved durability. The new coating is fully qualified for space in a more extreme environment than required by JPSS. The DNB Focal Plane Interface Electronics (FPIE) select resistors will be adjusted differently to address Low Gain Stage saturation providing improved anti-blooming performance. The SMWIR and Long-wave Infrared (LWIR) FPAs have improved alignment between the Microlens and IFAs to avoid an irregular Line Spread Function as seen on NPP. Recently, we implemented a flexure design into the LWIR and SMWIR bezel assemblies which will reduce thermal stresses on the IFA/Microlens assemblies increasing reliability.

The VIIRS J1 Cryoradiator, Mainframe, and Solar Diffuser Stability Monitor (SDSM) have changes to address lessons learned from NPP. The Cryoradiator Launch Locks were redesigned to address risks associated with thermal gradients due to solar exposure which exceeded design limits on NPOESS. The new design meets loading conditions under all required conditions and provides higher confidence of retaining the necessary preload. The NPP Cryoradiator Outgas Heaters caused predicted gradients during outgas that would exceed cryo design stress limits if the heaters were left on continuously. The NPP spacecraft addresses this concern by modulating heater power. JPSS will reduce the wattage on the outgas heaters to eliminate the problem. Cryoradiator Blankets were redesigned to eliminate door deployment interference with a cryoradiator blanket. To support improved cooling of the DNB during the warmer periods predicted for J1 orbit, the DNB Coldstrap has improved thermal impedance. The Mainframe design has some minor modifications which include an additional baffle internal to the mainframe to block earthshine on the blackbody reducing the need for a blanket. An access panel was added to allow FPA connector mate and demate after cryoradiator integration. The SDSM alignment process changed in the $\mathrm{J} 1$ integration and test procedures to characterize its performance over a larger range of solar angles. Recently, the JPSS team determined the most likely stray light paths which are causing the NPP DNB issues and will add light baffles and covers to the JPSS designs to protect against recurrence.

\subsubsection{ATMS}

The JPSS Phase Lock Oscillator (PLO) design was changed to a monolithic microwave integrated circuit (MMIC) to address parts obsolescence since NPP. No performance impacts are expected. The Local Oscillators (LO) were similarly redesigned to replace the obsolete Gunn diode based oscillators. The Receiver Front End (RFE) modules were redesigned for NPOESS and are performing well. The V-band intermediate frequency (IF) module output amplifiers were procured from a different supplier to the same original design to lower cost and reduce risk. The Receiver Power Supply (RPS) DC/DC Converters have an improved Local Oscillator design as well. The ATMS scan drive bearing retainers were redesigned using different materials from that on NPP to improve life expectancy and improve the reliability of the system. Parts obsolescence drove some design changes to the Digital Signal Processing (DSP) and 
Video Digitizer CCAs. Significant improvements have been made to the antenna and polarization tests conducted at the vendor. This knowledge will improve the calibration of the instrument. The NPP ATMS instrument is performing very well and JPSS expects similar or better performance as a result of the improvements and changes to the pre-launch characterization testing.

\subsubsection{CrIS}

The major change to CrIS for JPSS is the new one piece AlBeMet machined frame which replaced the 94-piece brazed AlBeMet Frame. This new frame eliminates the brazed joints. It has been delivered and tested. A redesigned Internal Calibration Target (ICT) will be flown on JPSS significantly improving emissivity and calibration performance; it has been built and tested. The NPP ICT was difficult to manufacture and presented a cost and performance challenge on JPSS. A simpler and more predictable design will be used on JPSS. As with VIIRS, CrIS will replace the 1394 FireWire interface with SpaceWire. For CrIS, the Spacewire implementation is a repackaged GOES-R design. Two circuit card types are to be replaced with improved versions if available in time to meet the $\mathrm{J} 1$ schedule. The board redesigns will increase reliability by removing the white wires and previously needed modifications. The Optical Bench on NPP was a 10-piece Brazed assembly; for JPSS it is a single piece, machined structure which will provide improved dynamic performance. The Vibration Isolation System baseplate and Back Bracket changed to improve its dynamic properties and manufacturability.

As part of an NPP corrective action for future builds, the FPGA software was rewritten to eliminate any possibility of instrument lockup or reset during operation as well as correct telemetry errors. This further eliminates a constraint on use of the 40. Frame diagnostic mode and a Signal Processor reset issue. Lastly, these FPGA code updates will correct some minor telemetry and servo math errors.

To address reliability concerns from the NPP Trace Width and 150V path Spacing Exoneration Review, the Processing Control Electronics (PCE) operational bus current path trace widths were redesigned. To correct an overshoot risk within the Control Electronics CCA, Interpoint converters were replaced with newer lot date coded parts which includes Interpoint design updates to provide feedback compensation and remove excess load capacitance. This turn-on overshoot was observed during the circuit card testing and tailoring process and is now corrected.

Changes to cables and a shift in the master clock frequency were made to improve the EMI/EMC performance.

\subsubsection{OMPS}

The JPSS-1 OMPS instrument consists of two major assemblies, the Main Electronics Box (MEB) and the Nadir Sensor. On NPP, OMPS included the Limb sensor. Due to the removal of this sensor from OMPS for JPSS-1, some electronics are no longer required. Several changes were made to remove these unused circuits in a manner that protected the integrity of the OMPS electronics function. The Limb Signal Processing and Timing and Clocks boards were replaced with Limb load boards which simulate the Limb electronics from a power loading perspective. Improvements were made to the Processor Interface board to implement boot select fly-back diodes and improve telemetry filtering. The Nadir Signal Processing board will have improved common mode noise rejection performance. The Nadir Timing and Clocks board was changed to address Charge Coupled Device (CCD) clock rail droop. The Low Voltage Power Supply was redesigned to address overshoot and transient performance. A Motor Driver board modification was made to move a diode addressing one of the OMPS waivers and provide Thermo-Electric Cooler (TEC) controller improvements. On the Motherboard a 1553 input transformer was modified to address noise concerns in the communications circuit. The MEB Chassis was modified to add an EMI filter to address EMI outages seen on NPP. Other changes include the addition of cable mounting brackets to simplify cable routing and a change to the survival heater thermostat wiring to address an OMPS waiver. There was a survival thermostat set-point change to address survival heater operation lessons learned on NPP. As described earlier, cPCI connectors have been replaced on the OMPS Backplanes, Processor Interface Boards, and SBCs to improve system reliability.

The JPSS Nadir Profiler primary mirror has an improved surface figure; housing thermostats have an added insulation pad; and the alignment cube has improved coating that address three waivers from NPP. On the structure, there are 
improved conductive tape grounding and additional grounding straps. The OMPS team added EMI blankets underneath the radiators to improve radiated susceptibility performance.

A new solar diffuser design has been tested and will be used on JPSS to increase the calibration margin over that of NPP. Also, the optical mounts have interface modifications to improve boresight stability. The team also added new settling tests which should reduce risk at the next level of integration. Flexures were added to the Total Column slit and the method of attachment was modified reducing stress and potential deformation over temperature. Based on lessons learned from NPP related to axial slippage, the Calibration Mechanism motor resolver shaft and gear attachment were redesigned to prevent such slippage on JPSS. Also a lesson learned from NPP, the radiator-conduction bar interface has a change in the indium gasket which will eliminate mode shifts. Several test improvements were made such as modifications to the polarization test, thermal vacuum test gradients and calibration source power supplies which will provide better pre-launch characterization data.

\subsubsection{CERES}

Several changes were made to the Flight Model 6 (FM6) CERES from that of FM5 on NPP. The Longwave (LW) channel filter bandpass was expanded over heritage by 38 microns to improve science processing and provide limited redundancy in the event of a total channel failure. The FM6 CERES changed the fabrication and coating of the Mirror Attenuator Mosaic (MAM) which is expected to improve on-orbit stability of the MAMs. A new Short Wave In-flight Calibration Source (SWICS) reference photodiode was implemented that is $>30$ times more radiation tolerant; in addition further protection was incorporated with the addition of more shielding on SWICS cover. A flight software update was made to correct a timestamp interpretation error identified during the CERES FM5 NPP integration.

\section{Current Instrument Issues and Status}

The JPSS instruments are in varying phases of development. CERES is nearly complete; ATMS is in its final stages of integration; while VIIRS and CrIS are integrating and testing subassemblies. As a result of the NPP on orbit operations and the JPSS instrument build, several issues have been identified and corrective actions implemented. This section will discuss some of the more significant issues being worked and the current instrument build status. VIIRS is discussed in greater detail in a separate paper.

\subsection{ATMS}

The ATMS instrument development had only one significant issue which affected schedule. The issue involved the 183 GHz G-Band mixers. Several of the mixers failed testing with most likely root cause being damage due to electrostatic discharge. A contributing factor to the failure of these extremely sensitive devices was their age and the materials used to manufacture them. The ATMS mixers were originally procured on the NPP project in the 2003 timeframe. The JPSS team had concerns with time dependent intermetallic effects between the wire bonds and the solder affecting the long term reliability of the mixers. As a result, new mixers were built by NGES to the same design as the NPP mixers. Those mixers were built, tested and are now being integrated into the ATMS instrument. Figure 2 below shows the development schedule of the instrument. We are forecasting a late 2013 completion of the instrument. Well ahead of the 2015 need date. 


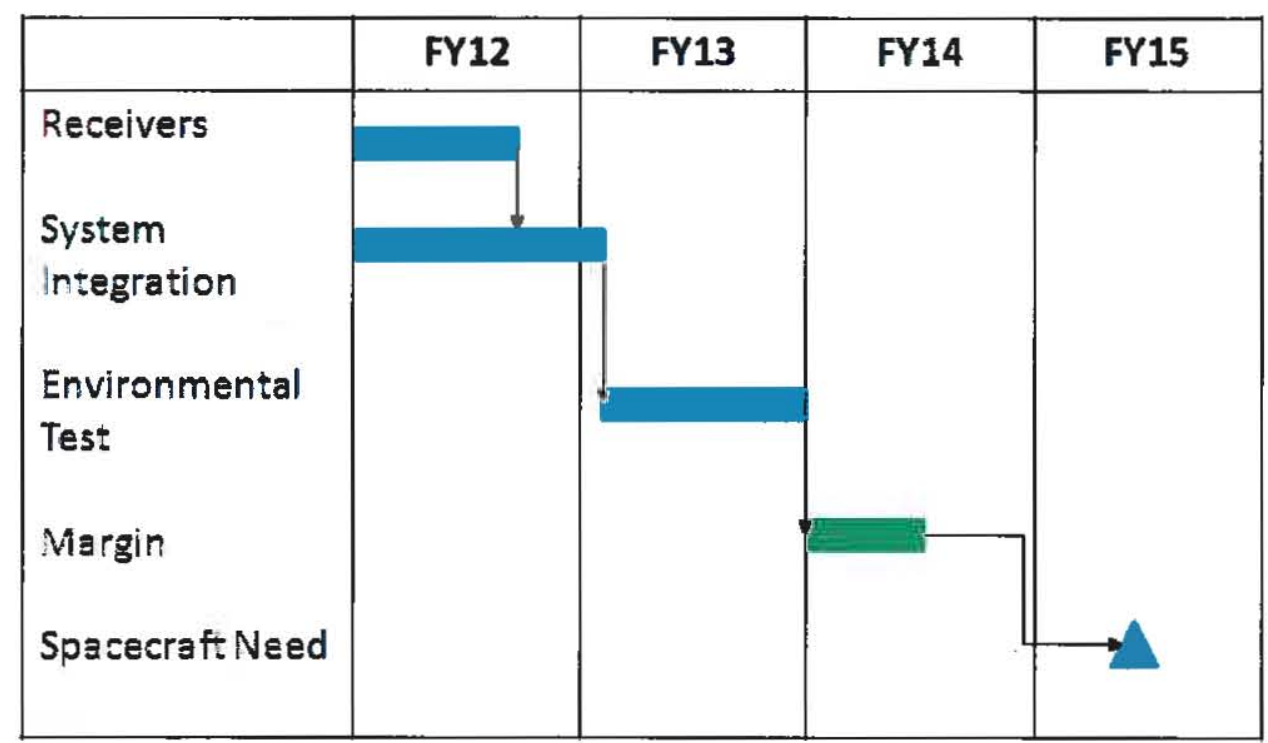

Figure 2. ATMS Schedule

\subsection{CrIS}

The CrIS instrument has also made significant progress toward delivery. The new frame has been designed, tested and is ready for integration. A spare frame is being built now and will be available for JPSS-2. The telescope has been built and delivered to ITT Exelis. It is undergoing its final tests prior to integration. The Flight Opto-Mechanical Assembly is complete. The new internal calibration target is complete and performing superior to that on NPP. The scene select module and critical co-registration testing is ongoing with good results. All focal planes have been tested and delivered. The Spacewire CCAs are the critical path, but are proceeding to plan. JPSS replaced the cPCI connectors on several CCAs and the motherboard to improve reliability all of which is complete and tested. A current issue which is nearing resolution is related to integration of the scene select module. Several low cost transducers were damaged during the installation due to their design, the tight tolerances and the tuning process. Design improvements are being implemented as are process improvements and new tooling to mitigate future risk of damage. Overall the CrIS instrument is on track for delivery in early 2014. 


\begin{tabular}{|c|c|c|c|c|}
\hline & FY12 & FY13 & FY14 & FY15 \\
\hline \multicolumn{5}{|l|}{ SpaceWire CCAs } \\
\hline \multicolumn{5}{|l|}{ CoolerCover } \\
\hline \multicolumn{5}{|l|}{ Vibration Isolation System } \\
\hline \multicolumn{5}{|l|}{ Earth Shield } \\
\hline \multicolumn{5}{|l|}{ Telescope } \\
\hline \multicolumn{5}{|l|}{ Frame } \\
\hline \multicolumn{5}{|l|}{ Internal Cal Target } \\
\hline \multicolumn{5}{|l|}{ Scene Select Module } \\
\hline \multicolumn{5}{|l|}{ Processing Control Electronics } \\
\hline \multicolumn{5}{|l|}{ Optical Module Assembly } \\
\hline \multirow{2}{*}{\multicolumn{5}{|c|}{ Co-Registration/SuperModule }} \\
\hline & & & & \\
\hline \\
\hline \multicolumn{5}{|l|}{ Environmental Test } \\
\hline \multicolumn{5}{|l|}{ Margin } \\
\hline Spacecraft Need & & & & \\
\hline
\end{tabular}

Figure 3. CrIS Schedule

\subsection{OMPS}

The JPSS OMPS instrument development was faced with only a couple significant challenges. The first was with the Single Board Computer (SBC) circuit cards. A parts corrosion issue on NPP led to the assessment that specific parts on the JPSS spares and flight circuit cards were suspect and at risk of future corrosion and failure. Additionally, the JPSS team evaluated the use of CCPI connectors for OMPS and determined the specific parts used were not desirable and posed a reliability risk. As a result, the SBC was redesigned to replace the suspect parts and replace the $\mathrm{cPCI}$ connectors with NASA approved Hypertronix connectors. The change to Hypertronix connectors also required a modification to the Backplane/Motherboard. In addition to the SBCs, the Processor Interface circuit cards were to be redesigned to replace their $\mathrm{cPCI}$ connectors. During that process it was determined that the printed wiring boards did not meet NASA standards. New boards were required and have been delivered. An issue with an oscillator was identified during testing of the PI board. Currently the team is working on the next steps for this circuit card. The SBCs are expected to be delivered by end of September 2012. The Nadir sensor was placed in storage in 2011 to reduce costs with plans to begin testing by the end of 2012 with a delivery for integration with the MEB by April 2013. The JPSS Project accelerated funding to the OMPS effort improving the delivery date by nine months. The OMPS instrument is on schedule for delivery in October 2013. Figure 4 below shows the OMPS schedule. 


\begin{tabular}{|l|c|c|c|c|}
\hline & FY12 & FY13 & FY14 & FY15 \\
\hline NadirSensor Tests & & & & \\
Flight Circuit Boards & & & & \\
New Processor interface CCA & & & \\
\cline { 2 - 4 } New Single Board Computer & & & \\
Chassis & & & \\
Instrument Integration & & & \\
Environmental Test & & & \\
Spacecraft Need & & & & \\
& & & & \\
\hline
\end{tabular}

Figure 4. OMPS Schedule

\subsection{VIIRS}

The JPSS VIIRS instrument has made significant progress. Nearly all of the circuit cards have been redesigned for J1 to incorporate the lessons learned from NPP (white wires) and increase both reliability and testability. The CCAs are complete, tested and being integrated into the Electronics Module (EM). The new circuit card test program proved incredibly more efficient than that seen on NPP reducing overall test time by more than half. On NPP, the circuit cards were a significant problem area which Raytheon has addressed for JPSS in both improved reliability and decreased production time. The RTA has improved mirrors and is built and tested. An effort is underway to develop the new Spacewire circuit cards and is on schedule. There have been some setbacks on the Aft Optics Assembly (AOA) which are being worked now. The Visible Near-Infrared (VisNIR) detector was found to have anomalies on the edges of the glass which were addressed and will not impact performance. The VisNIR Integrated Filter Assembly was reworked as a result of filter coating delamination, but is now delivered and tested. Also a straylight path was found and corrected in the VisNIR bezel shield design. The LWIR and SMWIR assemblies have had the most difficulty in completing. Both systems require a microlens and IFA to be bonded together with the bezel and aligned to the detectors. This bonded system design was found to have inherently high physical stresses over temperature whose reliability was dependent on the bonding process. After testing the systems, we found some failures in the bonds and have redesigned the system to add stress relief flexures. Those focal plane assemblies are in the final stages of integration and test. There completion and reintegration in September 2012 will be a major milestone for the VIIRS effort. Based on some data from the NPP mission, we are redesigning the single board computers (SBC) for JPSS to significantly increase their reliability and eliminate the need for periodic resets. We are addressing the NPP day-night band (DNB) straylight concerns through added baffling and coverage of potential light paths which should solve the problem for JPSS. The JPSS program has fully tested all its mirrors for tungsten contamination and do not anticipate any issues with contamination as seen on NPP. As can be seen in the figure below, most of the subsystems will be complete by the end of 2012 with instrument integration and test beginning in 2013. 


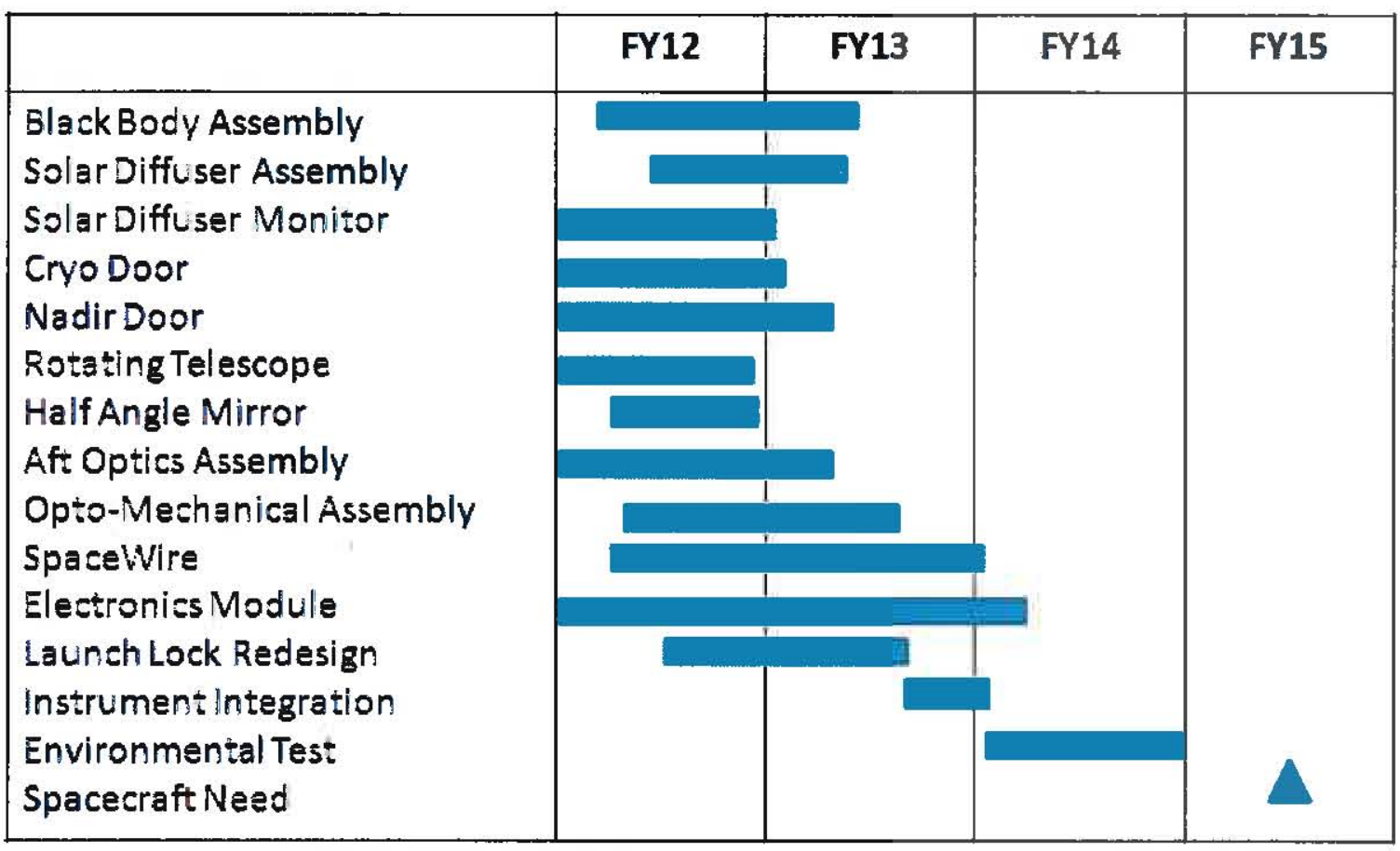

Figure 5. VIIRS Schedule

\subsection{CERES}

The CERES instrument is being built by Northrop Grumman Aerospace Systems (NGAS) under the management of NASA's Langley Research Center (LaRC). The instrument is completely built and tested; however a trending anomaly with the short wave calibration system was noted late in the calibration of the instrument. LaRC and NGAS are working to understand the root cause and will make necessary changes to the calibration lamps and photo-diode to ensure it meets specifications. We anticipate CERES completing its rework and testing by summer of 2013 for JPSS.

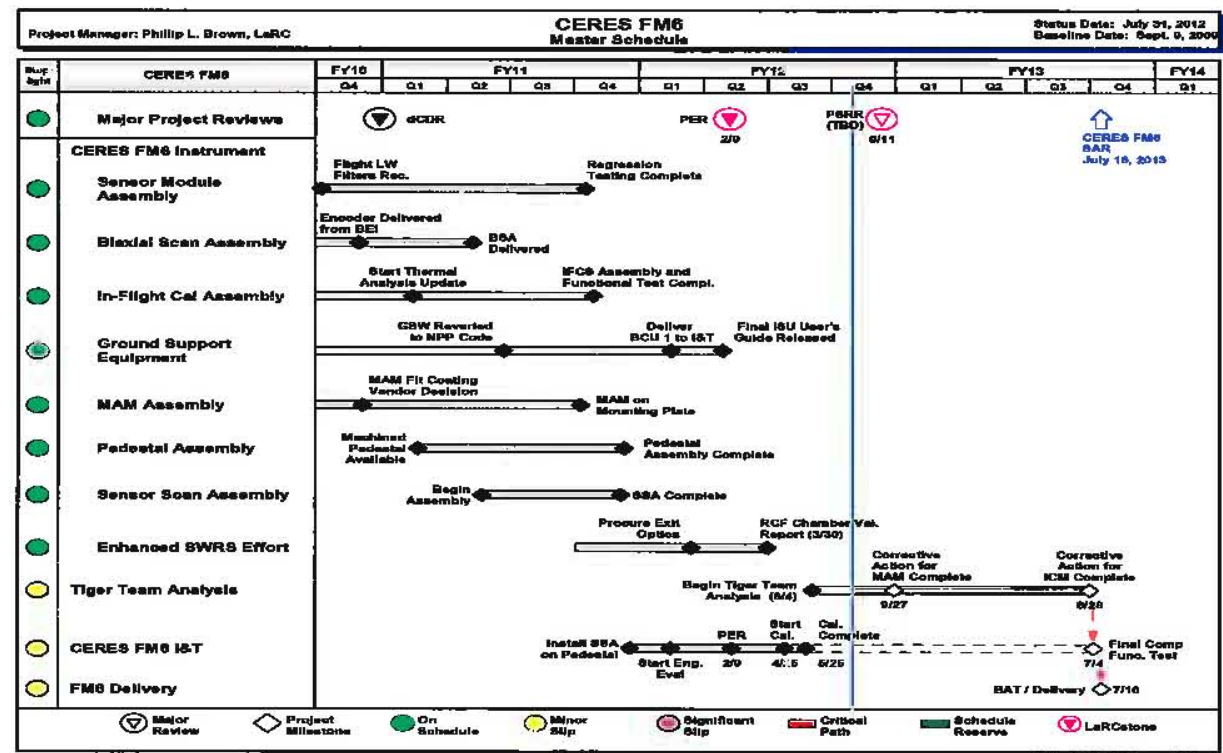

Figure 6. CERES Schedule 
